\title{
Dexterity-controlled Design Procedures
}

\author{
Federico Garrido ${ }^{1}$, Joost Meyer ${ }^{2}$ \\ ${ }^{1}$ TU Kaiserslautern ${ }^{2}$ RWTH Aachen \\ ${ }^{1}$ federgarrido@gmail.com²jmeyer@plastik.arch.rwth-aachen.de
}

This paper explores the development of design procedures in relationship to their digital proceedings, in order to interface human movement and parametric design procedures. The research studied the use of Leap Motion controller, a gesture recognition device using infrared sensors combined with time-based generative tools in Rhinoceros Grasshopper. A physical, artistic procedure was used as a reference to model a digital design procedure, including a series of parametric definitions combined with them in an attempt to produce complex three-dimensional designs in real time. In a later stage of this research, a modular, open source, digitizing arm was developed to capture hand movement and interact with an autonomous parametric definition, augmenting even more the range of applications of dexterity-based digital design. The challenge of this experimental investigation lies in the negotiation of the designer's needs for a complex yet open design process and the possibilities of defined soft-and hardware solutions.

Keywords: digital design, dexterity, parametric design, motion detection

\section{INTRODUCTION}

The utilization of gesture-based control is embedded on the digital architecture's history since Sutherland's first explorations in CAD technologies. Before the invention of the computer mouse and graphic interfaces, Sutherland developed a light pen that worked directly over the display and called the drawing commands using switches and knobs. Besides being a breakthrough at the time, Sutherland's Sketchpad was still related to the physical act of drawing, and the procedure he developed actually enhanced the user's drawing capabilities like zooming or copying.

The invention of the computer mouse and the command prompt eventually drove away these experimental devices in architecture, focusing on creat- ing methods for precise and quick drafting combining graphic interfaces, mouse movements and keyboard commands rather than a creative approach to form generation. Other artistic disciplines like illustrators or digital sculptors made use digital drafting equipment intensively.

For the last two decades and perhaps more, architectural research in the field of digital design tools focused on algorithmic tools and their derivatives: NURBS, scripting, parametric design, genetic algorithms, away from handmade form. Fuelled first by the popularization of personal computing and then by the advancements in digital fabrication, the center of attention was set on geometry: how to control it, how to represent it, how to manufacture it.

Scripting and parametric tools are key tools of 
this paradigm; complex geometrical compositions are generated and controlled by a series on intertwined values, variables and parameters. Form is created by repetition, translation, displacement, pressure, weight, self-organization, swarms and many other mathematically defined techniques. Control is the keyword: every geometrical aspect of the design is governed by a numeric value, a slider or a mathematical formula.

A common critique to this approach rises in regard to authorship and the role of the designer; there is a frequent sensation that the more (geometric) control we exercise, the more freedom we gain yet the authorial oversight seems to diminish. The designer usually takes the role of a breeder or a collector, selecting the best option out of many computergenerated variations.

This still is opposite to an analog design process which is, in most cases, a creative act. This act is accompanied by an immediate, often implicit reaction to the material and its properties. Throughout the digital design procedure most of the times there is a lack of material and time based response, a spontaneous feedback through the soft or hardware. With this research, we aim to contribute to fill this gap between a purely digital design methodology and the requirements of a real-time, hand-controlled, complex process.

On this regard, this paper explores how to generate complex geometries by using motion sensor devices in order to capture hand movement and incorporating it on a parametric definition. The purpose is to capture human movements, acceleration and dexterity and translate them to algorithmic values in order to generate and control detailed geometry.

The questions that this research proposes are: Which hidden design possibilities arise from capturing human movement into a design process? What is gained and what is lost on this translation procedure? And finally, is it possible to code these movements, automate and optimize such processes in order to reuse them?

\section{STATE OF THE ART}

The interest of this research is to capture human movement, either with optical or mechanical sensors and incorporate in a design process. For this matter we use a parametric design environment (Rhinoceros Grasshopper) and sensors like the Leap Motion infrared sensor and a self-developed device similar to a digitizing arm. By using these tools, it is possible not just to capture movement but also to augment in real time it in order to generate three-dimensional form.

There were several attempts to capture human movement and digitalize it in a design environment with a considerable amount of precision. These projects can be divided by the type of sensors they use and therefore, the type of movement they can capture. For example, the use of infrared cameras and body sensors like the Microsoft Kinect can capture the entire body with a relatively good resolution, however they cannot capture precise finger movement. On the other hand, devices like Leap Motion can detect and identify hand and fingers with considerable accuracy, yet the overall position of the hand is sometimes faulty.

Sketch furniture (Front Design 2006) is an interesting example of a design process based on body movement. On their project, an operator would freely sketch a three-dimensional line in space by moving his or her arms on the air, while infrared sensors capture the movement and design software converts the drawn lines into $3 \mathrm{~d}$ solid 'pipes'. On a second step, these $3 \mathrm{~d}$ bodies are manufactured with rapid prototyping techniques and can be used as furniture, like chairs or lamps. With this method the user can design tables, chairs and tube lamps by moving his hands, similar to drawing on the air. The Swedish studio uses an array of infrared cameras distributed on a room in order to produce the motion capture.

The 'L'Artisan Électronique' installation by Unfold (2010) uses laser sensors to capture the hand's position in order to generate vases by a rotational movement, similar to the ceramic ones created by a turning table. On this project, a linear laser is pro- 
jected against a surface and when it is intercepted by hand movement, the profile is captured by a camera and digitalized. The two-dimensional line is interpreted as the profile of the vase as it rotates, shaping its form in real time. The importance of this project for the research is that it operates by translating a physical artistic procedure (pottery turning) into a digital method of form making providing a real-time, visual feedback to the artist.

The 'Digital hammer' project by K. Hinton, also works on this direction but somehow indirectly. The intention of the researcher was to convert human movement into a software tool or modifier, functioning like a real hammer, but operating on a digital body. This way, when the artist hits a physical object with the sensor, the digital body is deformed. The artist developed a device and a software interface as an experimental "digital hand tool", intending to create an accessible tool for manipulating metal bodies within a digital platform, producing unique, unachievable pieces with other methods.

It is also important to state that on both cases, the sensor information is coded into a closed design system: the first one in a sketch-based 3D-pipe and the second one, a revolution-type solid. Both projects lack any sort of material constrains, and since the movement is unrestrained, there is no haptic feedback that can influence or limit the design, something that Hinton's digital hammer provides. On the case of Front's project, the artist moves the hand, arm and body in the air with no spatial reference other than his body and his own memory. Although they are not closed-source, both devices were specially created for that specific task and did not seem to be integrated into other design procedures.

On the other hand, there are special sensors which are commercially available and open-source, allowing designers to alter them and to include them into larger, more complex and more flexible design procedures.

Several research teams have developed gesturebased applications using commercial sensors like the Kinect (Microsoft), Leap Motion and the Wiimote tracker (Nintendo). The use of sensory devices such as Kinect and LeapMotion was also used to investigate complex hand sign recognition in real time (MARIN, 2014).

The research of motion detection sensors is also relevant to this research but our aim is to include them into a wider, more open design framework, such is the case of Rhinoceros Grasshopper. Grasshopper is a popular parametric design plug-in that works as a plug in on the Rhinoceros software, based on NURBS technology. Its extensive use in design and architectural applications make it an interesting choice to effectively interface gesture recognition within a design procedure.

Another interesting application of motion detection is made through the use of digitizing arms. They are often used to scan objects in three dimensions by locating points using an articulated arm. These arms often have three, four, five or more axes in order to accurately locate its tip in space.

Andrew Payne developed a 5-axis digitizing arm to control a 5-axis robot in real time using a similar technique, through the Grasshopper interface (Payne 2011). The digitizing arm works a scaled copy of the segments and articulations of a robot arm, this way the control is directed by the user and mimicked by the robot in real time using a FTDI chip as interface. On a later stage of this research, we designed a modular arm using Arduino microcontroller and angle sensors with the intention to create a modular, upgradable design.

\section{OBJECTIVES}

The intention of this research is to include hand motion and dexterity into a wider, flexible design methodology. Apart from sketch applications, the idea is to effectively use motion capture information to produce complex results, impossible to achieve by other means within a design environment. For this matter, we have designed several custom scripts or small programs that act as design procedures, performing a series of geometric operations using hand and finger movement as input data. 
Figure 1

Design research method
The idea of this study is to bring together the advantages of both approaches, manual and digital. Our research intends to study and mimic manual procedures performed with digital environment, aimed towards a better understanding of both types of procedures, finding their limitations and opportunities.

Instead of using the hand movement to draw lines freely like Front's Sketch Furniture project, our research intends to tracks hand and fingers and then mount a set of geometrical tools on top of them. This way, the digital procedure does not only consist on capturing hand movement but also includes stabilization algorithms, drawing presets and selfgenerating geometry.

On these terms, devices like Leap Motion have proved an interesting exploration tool but also evidenced a limited functionality in terms of precision and feedback, since they use an array of infrared cameras to detect fingers and articulations. The user has no physical feedback about where the hand or finger is in the three-dimensional space, nor a precise idea of what is he or she drawing in space. Unfold's project (Unfold 2010) deals with this issue partially by allowing the user to draw a flat line in space. This line is projected towards a plane and will be in turn be used as a revolving profile, limiting the drawing to a two-dimensional task. This simple geometric procedure marginally improves formal control and feedback.

The combination of new tools, which respond to manual dexterity (Grunwald 2016) and software instrumentalization aims to improve a digital workflow. We operate under the definition of dexterity as a degree of ease, speed and accuracy of human actions (Froehlich, and Drever 1983); this also includes a better awareness and understanding of spatial conditions and precise use of those tools throughout the design process. 
Figure 2

Burning shark, Joost

Meyer, 2017
For this reason, at the Faculty of Architecture, RWTH Aachen University, J.Meyer and F.Garrido have developed extensive experiments on these algorithms using sensors, of which first results were presented and discussed on the RCA Conference at TU Kaiserslautern in 2018 for the first time. At a later step the team designed and developed a 5 -axis digitizing arm in order to improve precision and add more design features to the drawing procedure.

\section{RESEARCH PROCEDURE}

The research consisted in translating dexterity-based procedures into a digital design environment, by gradually using sensory devices, micro-controllers and parametric design software (Fig. 1). As a first step, the manual method or procedure was analyzed, then, it was translated into a digital environment and finally a common framework was developed to include both "artistic" methodology and a digital, autonomous one. The intention is to produce novel design techniques that can benefit directly from the designer's skill and abilities, impossible to produce by standard digital means.

The first assignment of this research consisted on studying and analyzing a procedure, based on a material-related task. As a first example, we studied the artist Joost Meyer and his 'Shark' series. This group of sculptures was fabricated with metal wire rolled around a substructure on different sizes. They were selected because these works are procedurebased, they cannot be projected with precision since their final form depends on the execution of a task, influenced by materials and a shaping process.

Meyer first creates a wooden structure according to the shape and proportions of each particular fish and then proceeds to roll up metal wire (of different diameters) around the structure. The steel wire takes the form of the fish by adjusting itself more or less precisely to the shape of the wooden parts. Finally, the wooden structure is burned exposing the wire and some of the burnt remains.

The process is time consuming not only because of the size (some sharks are on a one to one scale) but also because of the rolling procedure using a continuous wire; the weight of the material, its resistance to bending, and elasticity difficult its manipulation (Fig. 2).

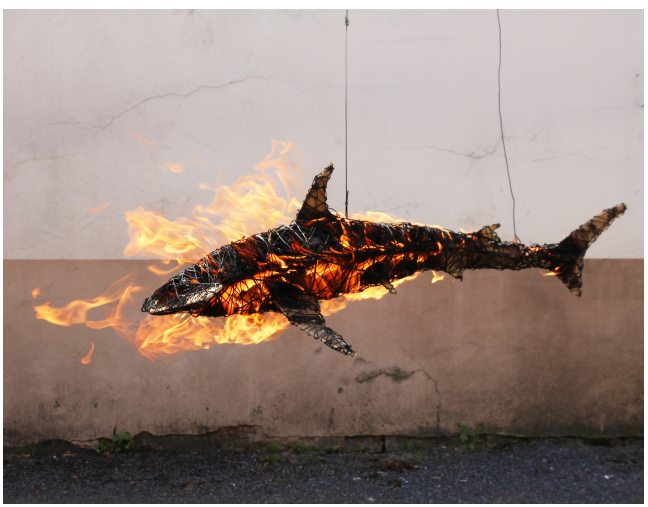

The purpose was to study the wire's movements, particularly in relation to the complex shark geometry. Sharks have a longitudinal shape which determines the main direction of the rolling movement. The presence of fins (dorsal, pectoral and caudal) forces Meyer to change the 'rolling' direction in order to precisely mimic each shape. Material properties like stiffness and ductility need procedure is based on dexterity, experience, craftmanship, and material knowledge.

A second task involved the study of Leap Motion, an input device originally designed for virtual and augmented reality applications. The device uses stereoscopic infrared cameras in order to track the movement of both hands; it can recognize fingers, palm orientation and some gestures such as pinching or grabbing. The integration between the Leap Motion controller and Grasshopper is made with two plug-ins, one is Primate (specifically designed for the controller) and Firefly (also used to integrate with other microcontrollers like Arduino). Thanks to this integration, Grasshopper can interpret the hand as three-dimensional geometry; the fingertips and finger joints as points, the hand palm as planes, and bones as lines. It is noteworthy that the Leap Motion 


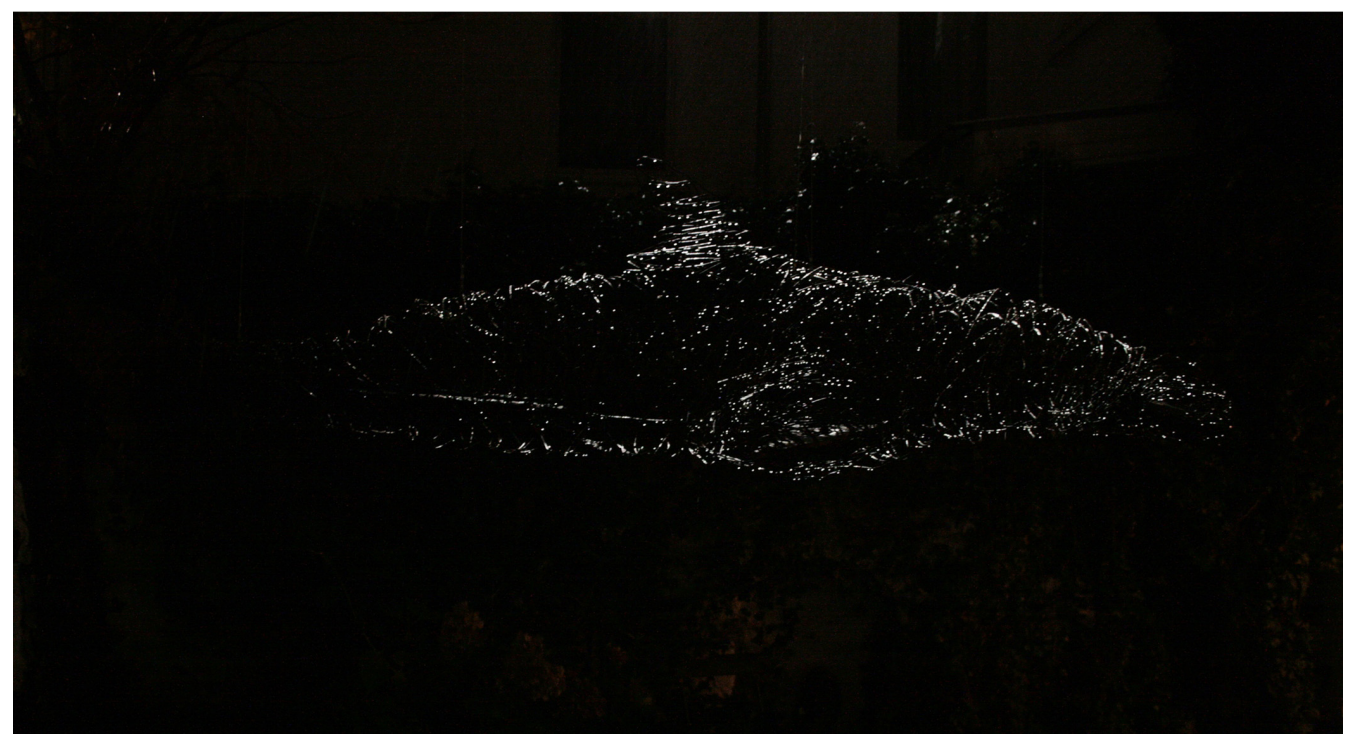

Figure 3

Shark sculpture by Joost Meyer

device was intentionally designed to be mounted on VR goggles following its movement, but it also works standing on a fixed base. Because of this, the result is that there is a range from which the device can capture fingers with relative precision; when the hands are moved further away from this range (which is around $50-70 \mathrm{~cm}$ ) the detection becomes imprecise. This limitation needed to be included on the design procedure.

The third phase consisted in the design of a parametric definition that identifies an index fingertip and uses it to draw in a three-dimensional environment. Leap Motion can capture all five fingers from each hand but for the purposes of this research, the use of more than one finger proved to be unnecessary. The definition is fairly straightforward as it captures the position of the index finger two hundred times per second, stamps a point on each location and then draws a line connecting them.

This phase was useful to test the controller and its capabilities, as the result is a doodle-like curve drawn in three-dimensional space. This result is also similar to the above-mentioned Sketch Furniture project, only on a smaller scale. Several iterations of this definition were tested, changing the sample rate and averaging algorithms in order to 'stabilize' the finger's position into a more fluid movement.

There are several problems from this approach since the capturing device is not one hundred percent accurate and sometimes, there is not direct line of sight between the sensor and each finger. There are a number ways by which these smoothing algorithms work; either by reducing the number of readings per second, or the 'stamps' on space, or by averaging the continuous stream of data.

By altering these parameters, the shaky lines began to smoothen into more NURBS-like curves. Here is yet another procedure from which we can smooth the result of the drawing. By generating a 0-degree NURBS curve, the connection between each point is direct, defining a polyline with multiple segments. If we increase this degree parameter, the component uses the points as 'weights' which pull the curve, resulting in a smoother curve. The higher the degree, 
Figure 4

Working in

Rhinoceros

environment with

mesh structures

and wiring

machine, F. Garrido

and J. Meyer, 2018

Figure 5

Dexterity based

form generation, $\mathrm{F}$.

Garrido and J.

Meyer, 2018

Figure 6

Human parametric design

collaboration for

form generation, $\mathrm{F}$.

Garrido and J.

Meyer, 2018

Figure 7

Shark evolution.

From hand drawing

to computer

generation the smoother is the result, but also, more imprecise, as the resulting curve avoids the points defined by the hand.

A digital design process involves the translation of material properties into digital parameters. We could theoretically use this geometric characteristic of NURBs curves as an analogy for material behavior. We used curve types and sample rates to emulate material behavior. To complete this material understanding, it would be necessary to consider and include manufacturing information into the process as well.

A number of combinations of these algorithms were tested in order to strike a balance between smoothness and precision. Several 3D-sketches were made with this procedure, however lacking any sort of haptic feedback, spatial reference or any other sort of guide apart from the visual, the result relied more on the user's dexterity than a robust, controlled drawing framework.

For this matter, we decided to imitate Meyer's procedure and work with a substrate, a base model to which the hand drawing could compare to. An organic mesh was inserted in the work environment, much like the shark's wooden structure in order to guide the user and his movements on the three-dimensional environment. This base mesh was scanned from a previous Meyer sculpture, remodeled, re-topologized and imported into the Rhinoceros environment (Fig. 4).

The final definition would use this base mesh as a support structure, projecting the hand drawn wire to it, adjusting the drawing lines to its shape, much like Meyer's wire. The precision of this projection can be altered along with the 'resistance' of the wire, allowing the exploration of even more aesthetical effects. The degree of which the hand drawn lines attach or detach from the base mesh can be calibrated in order to obtain figures more or less resembling to the original sculpture (Fig. 5). On this procedure, user's dexterity and the characteristics of the sensor are
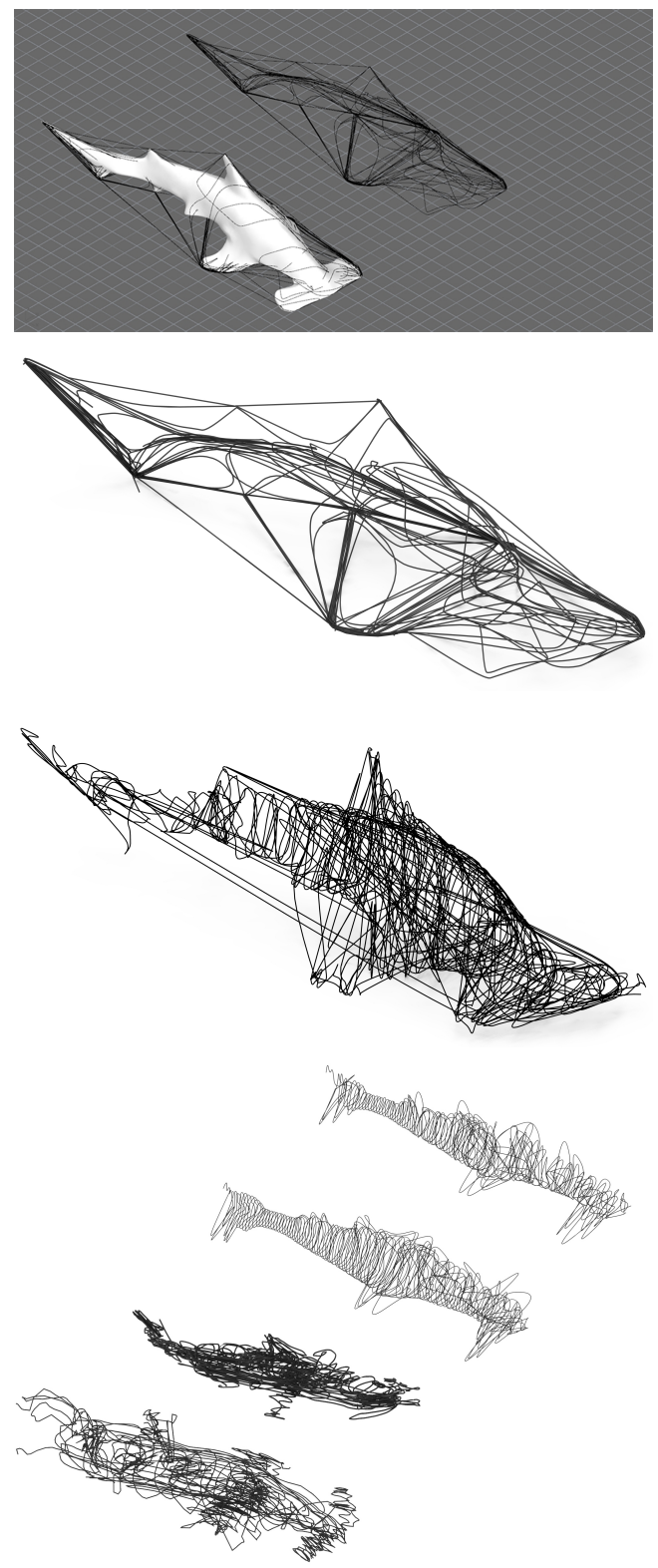
the keys to a successful design: direction of movement, acceleration and perception of the sensor may alter the design significantly.

The next step consisted in the creation of a definition that performs Meyer's procedure autonomously. The basic procedure is similar to the one previously described: a wire-rolling process that translates from one end of the fish structure to the other, creating a wire-cage structure mimicking it substrate. A time-based definition with movement controls such as speed, precision, and direction was implemented, operating either lineally (along an axis) or following a predetermined path. Similarly, the degree of precision with which the wire sticks to the substrate can be altered, along with several other factors like the speed of the machine or the curve's precision and degree.

Finally, a combination of the last two definitions was developed with the main characteristics of the in mind: on one hand, the creative possibilities of freehand modeling and on the other, the precision and versatility of computer generated procedures. This definition places a wire-bending procedure at the user's fingertip by using hand location to move the wire bending apparatus while the rolling movement is active. This way, the user can position the wire three-dimensionally and decide in which direction the wiring procedure is performed while the base mesh remains in place (Fig. 6).

This definition provides even more detail and creative possibilities since it allows the user to reorientate the wiring direction in order to adjust it to the form he wants to cover, which becomes particularly useful in areas like fins and other parts (Fig. 7). Similarly to other previous definitions, the lack of feedback and location makes the wiring process occasionally difficult. A similar definition was also developed, but this time, the user's hand moves the support mesh while the wiring machine stays in a fixed position.

The combination of hand movement and timebased, form-generating procedures unlocks many potential creative uses, for artists and designers as well. Another example of such methodologies is another experiment that uses three-dimensional blocks as a drawing material. This way, the user draws by adjoining $3 \mathrm{~d}$ shapes next to each other rather than a continuous line or pipe.

\section{HARDWARE DEVELOPMENT}

A later step of the research consisted in the development of a 'digitizing arm' device in order to capture movement with more precision. Using a mechanical device instead of an infrared one (such as the Leap Motion) brings several advantages such as improving precision and avoiding interruptions from other objects, light conditions or the identification of the hand itself. It also provides an interesting feedback since it requires the user to move a physical, articulated object in space in order to reach certain points and positions. The mechanical arm allows a basic spatial awareness of a different kind than just moving arms and fingers on the air.

The design of the arm is based on a 5 -axis robot, providing enough freedom of movement in space, without much mechanical resistance. Instead of actuators, on each articulation there are potentiometers or angle sensors, which are connected to a microcontroller communicating with the parametric software.

Using an open source microcontroller like Arduino allows multiple points of personalization and modification in a continuous prototyping process. For example, angle sensors can be replaced for more precise ones when they become available or the arm's proportions can be altered to fit certain design requirements. In fact, since the design is based on open source materials and modules, the digitizing arm can be scaled to any size and allow the attachment of different tooltips or other articulation configurations.

The device works by logging angle data from each articulation, recreating a digital version of the arm in Grasshopper in order to detect the tip of the digitizing tool. The necessary calculations translating angle information to three-dimensional position- 
Figure 8

5-axis digitizing arm by F. Garrido, 2019

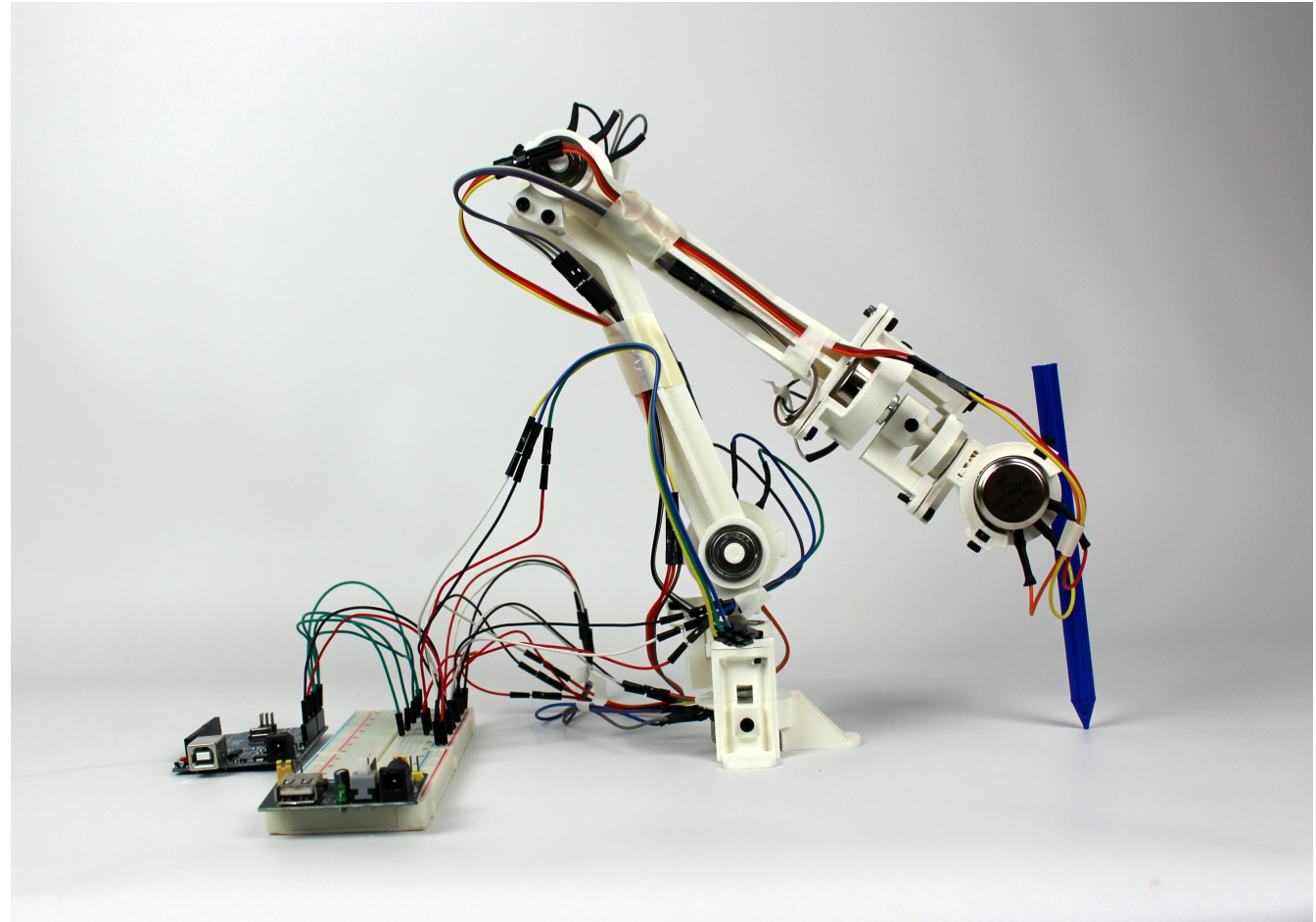

ing is made in the design software; this way, angles and positioning can be stored or modified in real time allowing even more personalization. In fact, the arm can also be used for example, for scanning three-dimensional objects by storing point portions in space or drawing isocurves. This is yet another opportunity to use capturing devices creatively.

The intention of the research is then to make design processes even more flexible both in software terms as well as with hardware components, where modularity and interchangeability are key concepts.

\section{CONCLUSIONS AND FUTURE STEPS}

The introduction of gesture-based control is embedded on the digital architecture's history since Sutherland's first explorations in CAD technologies using a prove communication between digital tools and designers through design processes and devices. While creating the wired silhouettes of a shark, new perspectives on creating form emerged as the negotiation between designer and scripter lead to an inspirational cooperation in the use of tools and a common design ambition.

The development of procedures and devices still faces the problem of haptic feedback. The development of the 5 -axis digitizing partially solved this issue providing spatial referencing. The use of open source hardware can improve on these solutions by adding for example, visual or haptic reactions triggered from digital data.

The popularization of alternative input devices light pen. This study is linked to other attempts to im- 
and microcontrollers eventually made possible this type of research again to a broader public, creating interesting projects as the aforementioned investigations. Despite the latest advancements, there is still room for improvement and new applications, as we particularly see it, regarding collaboration between digital processes and hand-controlled procedures. These procedures demand a new consciousness about fabrication process, combining traditional technique and digital ones in new hybrid ways of designing.

The expressive and aesthetic possibilities combined with machinic procedures and precise hand movement can generate detailed, intricate geometries, impossible to create with an either isolated method. Last to say is, that the multifaceted possibilities of digital methods need to be further explored. On the other hand, these developments, especially concerning usability and aspects of spatial awareness and haptic resonance to designers, are still in their infancy. We would like to contribute with this research to a better understanding of the interrelationship of designers and digital design methods.

\section{REFERENCES}

Adamson, G 2007, Thinking through craft, Berg Publishers, London

Fröhlich, W D and Drever, J 1983, Wörterbuch zur Psychologie, Deutscher Taschenbuch Verlag, München

Grunwald, M 2016, 'Haptik: Der handgreiflich区körperlicheZugang des Menschen zur Welt und zusichselbst', in Schmitz, T H (eds) 2016, Manifestationen im Entwurf: Design - Architektur - Ingenieurwesen, Transcript Verlag, Bielefeld, pp. 95-125

Hinton, K 2010, The Craft of Digital Toolin, Master's Thesis, Royal College of Art

Marin, G, Dominio, F and Zanuttigh, P 2014 'Hand gesture recognition with leap motion and kinect devices', 2014 IEEE International Conference on Image Processing (ICIP), Paris, pp. 1565-1569

Payne, A 2011 'A five-axis robotic motion controller for designers', Proceedings 31st Conference, Association for Computer-Aided Design in Architecture, Calgary I Banff, pp. 162-169

Warnier, C (eds) 2014, Printing things: visions and essentials for 3D printing, Gestalten, Berlin
Willis, K D D 2010 'Spatial sketch: bridging between movement \& fabrication', Proceedings of the fourth international conference on tangible, embedded, and embodied interaction, Cambridge, Massachusetts

[1] .http://unfold.be/pages/l-artisan-electronique

[2] http://www.frontdesign.se/sketch-furniture-perfor mance-design-project 